\title{
On the $\star$-product quantization and the Duflo map in three dimensions
}

\author{
Luigi Rosa and Patrizia Vitale \\ Dipartimento di Scienze Fisiche, Università di Napoli Federico II \\ and \\ INFN, Sezione di Napoli \\ Monte S. Angelo, Via Cinthia, 80126 Napoli, Italy \\ luigi.rosa@na.infn.it, patrizia.vitale@na.infn.it
}

\begin{abstract}
We analyze the $\star$-product induced on $\mathcal{F}\left(\mathbb{R}^{3}\right)$ by a suitable reduction of the Moyal product defined on $\mathcal{F}\left(\mathbb{R}^{4}\right)$. This is obtained through the identification $\mathbb{R}^{3} \simeq \mathfrak{g}^{*}$, with $\mathfrak{g}$ a threedimensional Lie algebra. We consider the $\mathfrak{s u}(2)$ case, exhibit a matrix basis and realize the algebra of functions on $\mathfrak{s u}(2)^{*}$ in such a basis. The relation to the Duflo map is discussed. As an application to quantum mechanics we compute the spectrum of the hydrogen atom.
\end{abstract}




\section{Introduction}

There has been recently a renewed interest in noncommutative structures on $\mathbb{R}^{3}$, mainly because of their occurrence in quantum gravity models [1], where $\mathbb{R}^{3}$, or copies of it, are identified with the dual algebra of the appropriate Lorentz group. In such framework $\star$ products are mainly introduced through a group Fourier transform (see for example [2, 3]), invoking compatibility with the group convolution. However, direct definitions are available in the literature, which don't rely on the existence of a group Fourier transform and have obvious calculational advantages. In view of applications to quantum field theory ([4]) we concentrate on this approach and refer to the cited literature for the other (cfr. [5] where a comparison is made in the last section).

Besides their appearence in the quantum gravity context, noncommutative structures on $\mathbb{R}^{3}$ are very interesting because of their emergence in the quantization of standard dynamical systems. To this, we shall discuss in some detail the application to the quantization of the hydrogen atom. As for their implications in quantum field theory, such that their renormalization properties and the UV/IR behaviour, which are quite different from noncommutative field theories on Moyal (hyper-)planes, they are analysed in [4].

The main object of this paper is to study in detail a family of star products introduced long ago in [6]. These products are obtained by a suitable reduction of the Moyal product on $\mathbb{R}^{4}$ on identifying the coordinate functions on $\mathbb{R}^{3}$ with one of the many subalgebras of the algebra of quadratic-linear functions on $\mathbb{R}^{4}$, the latter being isomorphic to the symplectic algebra $\mathfrak{s p}(4)$. This procedure induces on $\mathbb{R}^{3}$ a non-constant noncommutativity of Lie algebra type. We concentrate on the $\mathfrak{s u}(2)$ case, introduce a matrix basis and, in the spirit of deformation quantization, propose an application to the quantization of the hydrogen atom. At the same time we review a mathematically preferred quantization map, the Duflo isomorphism and show that our $\star$-product and the related quantization scheme are singled out among other possible definitions in that they agree with the Duflo map.

The paper is organised as follows. In Section 1 we review the family of $\star$ products introduced in [6] and we focus on the $S U(2)$ related $\star$-product. In Section 2, we define a matrix basis through a suitable reduction of the Moyal (hyper)-plane matrix basis [7, which has been so crucial for the renormalizability proof of the Grosse-Wulkenhaar model [8. The exhistence of a matrix basis for the three dimensional case is an important calculational tool, as it reduces the $\star$ product to a matrix product. In Section 3 after a short revision of the Duflo isomorphism we compute its value for the quadratic Casimir of $S U(2)$ and apply it to the quantization of the hydrogen atom, showing that it reproduces the correct spectrum if the whole symmetry of the system is taken into account. In Section 4 we perform the same calculation within the $\star$-quantization associated to the $\star$ product defined in Sections 1 and 2 and show that this $\star$ product is singled out among other possible definitions because of its agreement with the Duflo quantization. We conclude with some comments and perspectives. 


\section{Moyal induced star products on $\mathcal{F}\left(\mathbb{R}^{3}\right)$}

Let us consider the Moyal product [9, 10] defined on on $\mathcal{F}\left(\mathbb{R}^{4}\right) \simeq \mathcal{F}\left(\mathbb{C}^{2}\right)$. On using complex coordinates $\left(z^{a}, \bar{z}^{a}\right), a=1,2$ we have

$$
\left(f \star_{M} g\right)(z, \bar{z})=f(z, \bar{z}) \exp \left[\frac{\theta}{2}\left(\overleftarrow{\partial_{z}^{a}} \overrightarrow{\partial_{\bar{z}^{a}}}-\overleftarrow{\partial_{\bar{z}}} \overrightarrow{\partial_{z}^{a}}\right)\right] g(z, \bar{z})
$$

where the operator $\overleftarrow{\partial}$ (resp. $\vec{\partial}$ ) acts on the left (resp. on the right). It is well known that this product is induced through the Weyl quantization map [11] which, in two dimensions, associates to a function on the plane an operator according to:

$$
\hat{f}=\hat{\mathcal{W}}(f)=\frac{1}{(2 \pi)^{2}} \int \mathrm{d}^{2} z \hat{\Omega}(z, \bar{z}) f(z, \bar{z})
$$

where

$$
\hat{\Omega}(z, \bar{z})=\int \mathrm{d}^{2} \eta e^{-(\eta \bar{z}-\bar{\eta} z)} \mathrm{e}^{\theta\left(\eta a^{\dagger}-\bar{\eta} a\right)}
$$

is the so called quantizer and $a, a^{\dagger}$ are the usual (configuration space) creation and annihilation operators, with commutation relations

$$
\left[a, a^{\dagger}\right]=\theta
$$

The inverse map (the Wigner map [12]) is represented by:

$$
f(z, \bar{z})=\mathcal{W}^{-1}(\hat{f})=\operatorname{Tr}[\hat{f} \hat{\Gamma}(z, \bar{z})]
$$

with $\hat{\Gamma}(z, \bar{z})=\hat{\Omega}(z, \bar{z})$, meaning that the Weyl-Wigner quantization/dequantization procedure is self-dual (see [7, 13] for details).

The Moyal product is then defined as

$$
f \star_{M} g=\mathcal{W}^{-1}(\hat{\mathcal{W}}(f) \hat{\mathcal{W}}(g)) .
$$

The Weyl-Wigner-Moyal quantization scheme is the prototype of deformation quantization [14, where the algebra of operators is replaced by an algebra of functions with noncommutative product. From the latter expression it is not difficult (see for example [15]) to obtain integral expressions for the product, a few of which are collected in [6, Appendix]. The standard expression (1.1) is indeed an asymptotic expansion of the integral expressions [16]. It results

$$
\begin{aligned}
z \star_{M} \bar{z} & =z \cdot \bar{z}+\frac{\theta}{2} \\
\bar{z} \star_{M} z & =z \cdot \bar{z}-\frac{\theta}{2} .
\end{aligned}
$$

The dimension of the parameter $\theta$ depends on the physical meaning of the carrier space $\mathbb{R}^{2}$. In what follows we will choose the coordinate functions $z_{a}, \bar{z}_{a}$ and $\theta$ to be dimensionless. The generalization to the four dimensional case is straightforward. We recall that Weyl quantization associates to polynomial functions operators in the symmetric ordering, as opposed to other quantization schemes (see for example [17]). 
What is properly defined as the Moyal algebra is $\mathcal{M}_{\theta}:=\mathcal{M}_{L}\left(\mathbb{R}_{\theta}^{4}\right) \cap \mathcal{M}_{R}\left(\mathbb{R}_{\theta}^{4}\right)$ where $\mathcal{M}_{L}\left(\mathbb{R}_{\theta}^{4}\right)$, the left multiplier algebra, is defined as the subspace of tempered distributions that give rise to Schwartz functions when left multiplied by Schwartz functions; the right multiplier algebra $\mathcal{M}_{R}\left(\mathbb{R}_{\theta}^{4}\right)$ is analogously defined. For more details we refer to the appendix in [6] and references therein. In the present article we shall think of $\mathcal{M}_{\theta}$ as the algebra of $*$-polynomial functions in $z^{a}, \bar{z}^{a}$ properly completed. Its commutative limit, $\mathcal{F}\left(\mathbb{R}^{4}\right)$, is the commutative multiplier algebra $\mathcal{O}_{M}\left(\mathbb{R}^{4}\right)$, the algebra of smooth functions of polynomial growth on $\mathbb{R}^{4}$ in all derivatives [18].

The crucial step to obtain star products on $\mathcal{F}\left(\mathbb{R}^{3}\right)$, hence to deform $\mathcal{F}\left(\mathbb{R}^{3}\right)$ into a noncommutative algebra, is to identify $\mathbb{R}^{3}$ with the dual, $\mathfrak{g}^{*}$, of some chosen three dimensional Lie algebra $\mathfrak{g}$. This identification induces on $\mathcal{F}\left(\mathbb{R}^{3}\right)$ the Kirillov Poisson bracket, which, for coordinate functions reads

$$
\left\{x_{i}, x_{j}\right\}=c_{i j}^{k} x_{k}
$$

with $i=1, . ., 3$ and $c_{i j}^{k}$ the structure constants of $\mathfrak{g}$. On the other hand, all three-dimensional (Poisson) Lie algebras may be realized as subalgebras of the symplectic algebra $\mathfrak{s p}(4)$, which is classically realized as the Poisson algebra of quadratic-linear functions on $\mathbb{R}^{4}\left(\mathbb{C}^{2}\right.$ with our choices) with canonical Poisson bracket

$$
\left\{z^{a}, \bar{z}^{b}\right\}=\mathrm{i} \text {. }
$$

It is then possible to find quadratic-linear functions

$$
x_{i}=x_{i}\left(z^{a}, \bar{z}^{a}\right)
$$

which obey (1.8). This is nothing but the classical counterpart of the Jordan-Schwinger map realization of Lie algebra generators in terms of creation and annihilation operators [19]. Then one can show [6] that these Poisson subalgebras are also Moyal subalgebras, that is

$$
x_{i}\left(z^{a}, \bar{z}^{a}\right) \star_{M} x_{j}\left(z^{a}, \bar{z}^{a}\right)-x_{j}\left(z^{a}, \bar{z}^{a}\right) \star_{M} x_{i}\left(z^{a}, \bar{z}^{a}\right)=\lambda c_{i j}^{k} x_{k}\left(z^{a}, \bar{z}^{a}\right)
$$

where the noncommutative parameter $\lambda$ depends on $\theta$ and shall be adjusted according to the physical dimension of the coordinate functions $x_{i}$. Occasionally we shall indicate with $\mathbb{R}_{\lambda}^{3}$ the noncommutative algebra $\left(\mathcal{F}\left(\mathbb{R}^{3}\right), \star\right)$. Eq. (1.11) induces a star product on polynomial functions on $\mathbb{R}^{3}$ generated by the coordinate functions $x_{i}$, which may be expressed in closed form in terms of differential operators on $\mathbb{R}^{3}$. For details we refer to [6] where all products are classified. Here we will consider quadratic realizations of the kind

$$
\pi^{*}\left(x_{\mu}\right)=\kappa \bar{z}^{a} e_{\mu}^{a b} z^{b}, \quad \mu=0, . ., 3
$$

with $e_{i}=\frac{1}{2} \sigma_{i}, i=1, . ., 3$ are the $S U(2)$ generators and $\sigma_{i}$ are the Pauli matrices, while $e_{0}=\frac{1}{2} \mathbf{1}$. Here we have explicitly indicated the pull-back map $\pi^{*}: \mathcal{F}\left(\mathbb{R}^{3}\right) \mapsto \mathcal{F}\left(\mathbb{R}^{4}\right)$. We will shall omit it in the following, unless necessary. $\kappa$ is some possibly dimensional constant such that $\lambda=\kappa \theta$. Notice that

$$
x_{0}^{2}=\sum_{i} x_{i}^{2}
$$

It is possible to show that 6 ]

$$
\left(x_{i} \star \phi\right)(x)=\left\{x_{i}-i \frac{\lambda}{2} \epsilon_{i j k} x_{j} \partial_{k}-\frac{\lambda^{2}}{8}\left[(1+x \cdot \partial) \partial_{i}-\frac{1}{2} x_{i} \partial \cdot \partial\right]\right\} \phi(x)
$$


which implies for coordinate functions

$$
\begin{gathered}
x_{i} \star x_{j}=x_{i} \cdot x_{j}+i \frac{\lambda}{2} \epsilon_{i j k} x_{k}-\frac{\lambda^{2}}{8} \delta_{i j} \\
x_{i}, \star x_{j}-x_{j} \star x_{i}=i \lambda \epsilon_{i j k} x_{k}
\end{gathered}
$$

where we have indicated with $\star$ the induced star product. Moreover we have

$$
\left[x_{0}, \phi\left(x_{i}\right)\right]_{\star}=0
$$

That is, $x_{0}$ lies in the center of the algebra $\mathbb{R}_{\lambda}^{3}$, so that the latter may be equivalently defined as the commutant of $x_{0}$.

It is possible to use other star products on $\mathbb{R}^{4}$ such that the polynomial algebra generated by the coordinate functions in Eq. (1.12) is still a subalgebra with the same $\star$-commutator. See for example [20] where the Voros product has been used. This corresponds to the choice of normal ordering at the level of $\star$ quantization in $\mathbb{R}^{4}$. For applications of the Voros and other translation-invariant $\star$ products to 4 -dimensional quantum field theory (QFT) see for example [21], 22], 23]. While these products yield the same $\star$ commutator between coordinates, they might have important consequences which will be discussed in the last section.

The expression (1.14) which was derived in [6] for the star product in $\mathbb{R}_{\lambda}^{3}$ is practically difficult to use when we need to extend it to the product of two generic functions. In next section we shall derive a matrix basis for $\mathbb{R}_{\lambda}^{3}$ which makes it much easier to compute the $\star$-product. We shall see that it reduces the $\star$ product (1.14) to matrix multiplication.

\section{$2 \quad$ Matrix formulation of $\mathbb{R}_{\lambda}^{3}$}

In this section we derive a matrix formulation of $\mathbb{R}_{\lambda}^{3}$ which is based on a suitable reduction of the matrix formulation of the Moyal space $\mathbb{R}_{\theta}^{4}$ first introduced by Gracia-Bondia and Varilly in [7]. We also discuss the meaning of the new matrix basis in connection to the $\star$-quantization of the angular momentum and to the Wigner functions associated to the density operator of angular momentum states. To this, let us first review the derivation in [7] for the two and four dimensional case.

\subsection{The matrix basis of $\mathbb{R}_{\theta}^{2 n}$}

To a function on $\mathbb{R}^{2}$ we associate via the Weyl map (1.2) the operator

$$
\phi(z, \bar{z}) \rightarrow \hat{\phi}\left(a, a^{\dagger}\right) .
$$

This may be expanded into symmetric ordered powers of $a, a^{\dagger}$

$$
\hat{\phi}=\sum_{p, q} \tilde{\phi}_{p q}: a^{\dagger} a^{q}:
$$

with : : denoting the symmetric ordering. On using the number basis

$$
a^{\dagger} a|n\rangle=n|n\rangle
$$


together with

$$
a|n\rangle=\sqrt{n \theta}|n-1\rangle \quad a^{\dagger}|n\rangle=\sqrt{n+1) \theta}|n+1\rangle
$$

we may rewrite (2.19) as

$$
\hat{\phi}=\sum_{p, q} \phi_{p q}|p\rangle\langle q|
$$

with $\tilde{\phi}_{p q}, \phi_{k l}$ related by a change of basis

$$
\phi_{l k}=\sum_{q=0}^{\min (l, k)} \tilde{\phi}_{l-q, k-q} \frac{\sqrt{l ! k ! \theta^{l+k}}}{\theta^{q} q !}
$$

On applying the Wigner map we obtain a function in the noncommutative Moyal algebra

$$
\phi(z, \bar{z})=\sum_{p, q} \phi_{p q} f_{p q}(z, \bar{z})
$$

with

$$
f_{p q}(z, \bar{z})=\mathcal{W}^{-1}(|p\rangle\langle q|)=\frac{1}{\sqrt{p ! q ! \theta^{p q}}} \bar{z}^{p} \star_{M} f_{00} \star_{M} z^{q}
$$

and

$$
f_{00}(\bar{z}, z)=2 \exp (-2 \bar{z} z / \theta) .
$$

This Gaussian is idempotent w.r.t. the Moyal star product as it satisfies the condition

$$
f_{00} \star_{M} f_{00}(\bar{z}, z)=f_{00}(\bar{z}, z)
$$

Let us recall that, when qualifying $\mathbb{R}^{2}$ as the phase space of 1-dimensional systems, the basis functions $f_{p q}(z, \bar{z})$ in the $\star$-quantization scheme correspond exactly to the Wigner functions associated to the density operator of the quantum oscillator states.

The basis elements $f_{p q}(z, \bar{z})$ may be seen to obey

$$
f_{p q} \star_{M} f_{k l}=\delta_{q k} f_{p l}
$$

either by direct calculation, on using (2.27), or by observing that, by definition

$$
f_{p q} \star_{M} f_{k l}=\mathcal{W}^{-1}(|p\rangle\langle q \mid k\rangle\langle l|) .
$$

Moreover

$$
\int \mathrm{d}^{2} z f_{p q}(z, \bar{z})=2 \pi \theta \delta_{p q}
$$

The extension to $\mathbb{R}_{\theta}^{4}$ is straightforward. We have

$$
\phi\left(z_{a}, \bar{z}_{a}\right)=\sum_{\vec{p} \vec{q}} \phi_{\vec{p} q} f_{\vec{p} q}\left(z_{a}, \bar{z}_{a}\right)
$$

with $a=1,2, \vec{p}=\left(p_{1}, p_{2}\right)$ and

$$
f_{\vec{p} \vec{q}}\left(z_{a}, \bar{z}_{a}\right)=\mathcal{W}^{-1}\left(\left|p_{1} p_{2}\right\rangle\left\langle q_{1} q_{2}\right|\right)=f_{p_{1}, q_{1}}\left(z_{1}, \bar{z}_{1}\right) \cdot f_{p_{2}, q_{2}}\left(z_{2}, \bar{z}_{2}\right) .
$$

In order to describe elements of $\mathbb{R}_{\theta}^{2}$ (resp. $\mathbb{R}_{\theta}^{4}$ ), the sequences $\left\{\phi_{p q}\right\}$ (resp. $\left\{\phi_{\vec{p} q}\right\}$ ) have to be of rapid decay [7]. 


\section{$2.2 \quad$ The matrix basis of $\mathbb{R}_{\lambda}^{3}$}

In order to obtain a matrix basis in three dimensions, compatible with the product (1.14), we resort to the Schwinger-Jordan realization of the $\mathfrak{s u}(2)$ Lie algebra in terms of creation and annihilation operators. We observe that the eigenvalues of the number operators $\hat{N}_{1}=a_{1}^{\dagger} a_{1}$, $\hat{N}_{2}=a_{2}^{\dagger} a_{2}$, say $p_{1}, p_{2}$, are related to the eigenvalues of $\hat{\mathbf{X}}^{2}, \hat{X}_{3}$, respectively $j(j+1)$ and $m$, by

$$
p_{1}+p_{2}=2 j \quad p_{1}-p_{2}=2 m
$$

with $p_{i} \in \mathbb{N}, j \in \mathbb{N} / 2,-j \leq m \leq j$, so to have

$$
\left|p_{1} p_{2}\right\rangle=|j+m, j-m\rangle \equiv|j m\rangle=\frac{\left(a_{1}^{\dagger}\right)^{j+m}\left(a_{2}\right)^{j-m}}{\sqrt{(j+m) !(j-m) !}}|00\rangle
$$

where $\hat{X}_{i}, i=1, . ., 3$ are the standard angular momentum operators representing the $\mathfrak{s u}(2)$ Lie algebra in terms of selfadjoint operators on the Hilbert space spanned by $|j, m\rangle$. Then we may relabel the matrix basis of $\mathbb{R}_{\theta}^{4}$, Eq. (2.32) as $f_{m \tilde{m}}^{j \tilde{\jmath}}$, so to have

$$
\phi\left(z_{a}, \bar{z}_{a}\right)=\sum_{j \tilde{\mathrm{j}} \in \mathbb{N} / 2} \sum_{m=-j}^{j} \sum_{\tilde{m}=-\tilde{\mathrm{j}}}^{\tilde{\mathrm{j}}} \phi_{m \tilde{m}}^{j \tilde{\mathrm{j}}} f_{m \tilde{m}}^{j \tilde{\mathrm{j}}}\left(z_{a}, \bar{z}_{a}\right)
$$

We further observe that, for $\phi$ to be in the subalgebra $\mathbb{R}_{\lambda}^{3}$ we must impose $j=\tilde{\mathrm{j}}$. To this it suffices to compute

$$
x_{0} \star f_{m \tilde{m}}^{j \tilde{\mathrm{j}}}-f_{m \tilde{m}}^{j \tilde{\mathrm{j}}} \star x_{0}=\lambda(j-\tilde{\mathrm{\jmath}}) f_{m \tilde{m}}^{j \tilde{\mathrm{j}}}
$$

and remember that $\mathbb{R}_{\lambda}^{3}$ may be alternatively defined as the $\star$-commutant of $x_{0}$. This requires

$$
j=\tilde{\mathrm{j}}
$$

We have then

$$
\phi\left(x_{i}\right)=\sum_{j} \sum_{m, \tilde{m}=-j}^{j} \phi_{m \tilde{m}}^{j} v_{m \tilde{m}}^{j}
$$

with

$$
v_{m \tilde{m}}^{j}:=f_{m \tilde{m}}^{j j}=\frac{\bar{z}_{1}^{j+m} \star_{M} f_{00}\left(\bar{z}_{1}, z_{1}\right) \star_{M} z_{1}^{j+\tilde{m}} \bar{z}_{2}^{j-m} \star_{M} f_{00}\left(\bar{z}_{2}, z_{2}\right) \star_{M} z_{2}^{j-\tilde{m}}}{\sqrt{(j+m) !(j-m) !(j+\tilde{m}) !(j-\tilde{m}) ! \theta^{4 j}}}
$$

It is useful to recall its expression in terms of the Wigner map. We have

$$
v_{m \tilde{m}}^{j}=\mathcal{W}^{-1}(|j m\rangle j \tilde{m} \mid)
$$

In view of the application to Quantum Mechanics one can recognize this as the Wigner functions of the angular momentum eigenstates, that is the Wigner symbols associated to the density operator (cfr. for example [24])* The orthogonality property now reads

$$
v_{m \tilde{m}}^{j} \star v_{n \tilde{n}}^{\tilde{\jmath}}=\delta^{j \tilde{j}} \delta_{\tilde{m} n} v_{m \tilde{n}}
$$

\footnotetext{
* See also [25] where symbols for the angular momentum states are introduced in a different $\star-$ quantization scheme, the so called tomographic or probability representation of quantum mechanics [26, 27]).
} 
As for the normalization we have

$$
\int \mathrm{d}^{2} z_{1} \mathrm{~d}^{2} z_{2} v_{m \tilde{m}}^{j}(z, \bar{z})=4 \pi^{2} \theta^{2} \delta_{m \tilde{m}}
$$

The star product in $\mathbb{R}_{\lambda}^{3}$ becomes a matrix product

$$
\phi \star \psi(x)=\sum \phi_{m_{1} \tilde{m}_{1}}^{j_{1}} \phi_{m_{2} \tilde{m}_{2}}^{j_{2}} v_{m_{1} \tilde{m}_{1}}^{j_{1}} \star v_{m_{2} \tilde{m}_{2}}^{j_{2}}=\sum \phi_{m_{1} \tilde{m}_{1}}^{j_{1}} \phi_{m_{2} \tilde{m}_{2}}^{j_{2}} v_{m_{1} \tilde{m}_{2}}^{j_{1}} \delta^{j^{1} j^{2}} \delta_{\tilde{m}_{1} m_{2}}
$$

while the integral may be defined through the pullback to $\mathbb{R}_{\theta}^{4}$

$$
\int_{\mathbb{R}_{\lambda}^{3}} \phi \star \psi:=\kappa^{2} \int_{\mathbb{R}_{\theta}^{4}} \pi^{\star}(\phi) \star_{M} \pi^{*}(\psi)=4 \pi^{2} \lambda^{2} \operatorname{Tr} \Phi \Psi
$$

hence becoming a trace

In analogy with the present derivation, the matrix basis adapted to the Voros product can be found in [29], and it is reduced to 3 dimensions in [4] where applications to 3d QFT are studied.

\subsection{The $\star$-quantization of the the $S U(2)$ generators in the matrix basis}

For further application to the quantization of the hydrogen atom let us work out in detail the realization of the $S U(2)$ generators in the matrix basis. On using the expression of the generators in terms of $\bar{z}_{a}, z_{a}$ and the basis transformations (2.23) we find their expression in the matrix basis

$$
\begin{aligned}
& x_{+}=\kappa \bar{z}_{1} z_{2}=\lambda \sum_{j, m} \sqrt{(j+m)(j-m+1)} v_{m m-1}^{j} \\
& x_{-}=\kappa \bar{z}_{2} z_{1}=\lambda \sum_{j, m} \sqrt{(j-m)(j+m+1)} v_{m m+1}^{j} \\
& x_{3}=\frac{\kappa}{2}\left(\bar{z}_{1} z_{1}-\bar{z}_{2} z_{2}\right)=\lambda \sum_{j, m} m v_{m m}^{j} \\
& x_{0}=\frac{\kappa}{2}\left(\bar{z}_{1} z_{1}+\bar{z}_{2} z_{2}\right)=\lambda \sum_{j, m}\left(j+\frac{1}{2}\right) v_{m m}^{j}
\end{aligned}
$$

were we have introduced

$$
x_{ \pm}:=x_{1} \pm i x_{2}
$$

This yields

$$
\begin{array}{ll}
x_{+} \star v_{m \tilde{m}}^{j}=\lambda \sqrt{(j+m+1)(j-m)} v_{m+1 \tilde{m}}^{j} & v_{m \tilde{m}}^{j} \star x_{+}=\lambda \sqrt{(j-\tilde{m}+1)(j+\tilde{m})} v_{m \tilde{m}-1}^{j} \\
x_{-} \star v_{m \tilde{m}}^{j}=\lambda \sqrt{(j-m+1)(j+m)} v_{m-1 \tilde{m}}^{j} & v_{m \tilde{m}}^{j} \star x_{-}=\lambda \sqrt{(j+\tilde{m}+1)(j-\tilde{m})} v_{m \tilde{m}+1}^{j} \\
x_{3} \star v_{m \tilde{m}}^{j}=\lambda m v_{m \tilde{m}}^{j} & v_{m \tilde{m}}^{j} \star x_{3}=\lambda \tilde{m} v_{m \tilde{m}}^{j} \\
x_{0} \star v_{m \tilde{m}}^{j}=\lambda\left(j+\frac{1}{2}\right) v_{m \tilde{m}}^{j} & v_{m \tilde{m}}^{j} \star x_{0}=\lambda\left(j+\frac{1}{2}\right) v_{m \tilde{m}}^{j}
\end{array}
$$

\footnotetext{
${ }^{\dagger}$ If we were to perform our analysis in the coordinate basis, without recurring to the matrix basis, we should use a differential calculus adapted to $\mathbb{R}_{\lambda}^{3}$ as the one introduced in [28] .
} 
The same result could have been easily obtained on using directly the expression of the basis elements $v_{m \tilde{m}}^{j}$ and of the coordinates variables as functions on $\mathbb{R}^{4}$ and using the Moyal product. We have then

$$
x_{0} \star x_{0} \star v_{m \tilde{m}}^{j}=\lambda^{2}\left(j+\frac{1}{2}\right)^{2} v_{m \tilde{m}}^{j}
$$

which is different from

$$
\left(\frac{1}{2}\left(x_{-} \star x_{+}+x_{+} \star x_{-}\right)+x_{3} \star x_{3}\right) v_{m \tilde{m}}^{j}=\lambda^{2} j(j+1) v_{m \tilde{m}}^{j}
$$

\section{The Duflo map}

The Duflo isomorphism [30] first appeared in Lie theory and representation theory. For a detailed and pedagogical review we refer to [31. It is an algebra isomorphism between invariant polynomials of a Lie algebra and the center of its universal enveloping algebra. Kontsevich 32. later refined Duflo's result in the framework of deformation quantization.

Since the fundamental results by Harish-Chandra and others, it is now well understood that the algebra of invariant polynomials on the dual of a Lie algebra of a particular type (solvable, synple or nilpotent) is isomorphic to the center of the corresponding universal enveloping algebra. This fact was generalized to an arbitrary finite-dimensional real Lie algebra by Duflo in [30]. This isomorphism is called the Duflo isomorphism and it is obtained on composing the PoincaréBirkhoff-Witt isomorphism (which is only an isomorphism at the level of vector spaces) with an automorphism of the space of polynomials.

Because of its very definition the Duflo is a mathematically preferred quantization map, although it is often objected in the physics literature that its application to physics is ambigous as it doesn't reproduce the correct energy spectrum for well known problems in quantum mechanics like the quantization of the hydrogen atom Hereafter we will first derive the map for the quadratic Casimir of the rotation group and then apply it precisely to the hydrogen atom showing that we obtain the correct spectrum if the whole $S O(4)$ invariance of the system is taken into account.

Let us indicate with $S(\mathfrak{g})$ the symmetric algebra over the Lie algebra $\mathfrak{g}$. This may be identified with the polynomial functions of $S(\mathfrak{g})^{*}$. This is isomorphic to the universal enveloping algebra $U(\mathfrak{g})$ as a vector space. Let us consider the subalgebra of $\operatorname{ad}_{\mathfrak{g}}$ invariant polynomials, $S(\mathfrak{g})^{\mathfrak{g}}$ and let us define the Poincaré-Birkhoff-Witt map

$$
\begin{gathered}
I_{P B W}: S(\mathfrak{g})^{\mathfrak{g}} \rightarrow \mathcal{Z}(U(\mathfrak{g})) \\
I_{P B W}\left(x_{1}, \ldots x_{n}\right)=\frac{1}{n !} \sum_{\sigma(p) \in \Pi(n)} x_{\sigma(1)} . . x_{\sigma(n)}
\end{gathered}
$$

with $\mathcal{Z}(U(\mathfrak{g}))$ the center of $U(\mathfrak{g})$. This is an isomorphism of vector spaces but not in general an isomorphism of algebras. Generalising previous results of Haris-Chandra to all finite dimensional Lie algebras Duflo proved in [30] that it could be extended to an algebra isomorphism. Upon defining

$$
j^{\frac{1}{2}}(x):=\operatorname{det}^{\frac{1}{2}}\left[\frac{\sinh \frac{1}{2} \operatorname{ad} x}{\frac{1}{2} \operatorname{ad} x}\right]
$$

\footnotetext{
‡For applications to Quantum Gravity see [2, 33.
} 
the Duflo isomorphism

$$
\chi_{D}: S(\mathfrak{g})^{\mathfrak{g}} \rightarrow \mathcal{Z}(U(\mathfrak{g}))
$$

is proven to be

$$
\chi_{D}=I_{P B W} \circ j^{\frac{1}{2}}(\partial)
$$

where

$$
\partial:=\tau_{i} \frac{\partial}{\partial x^{i}}
$$

$\tau_{i}$ are the generators of the Lie algebra in the adjoint representation and $\partial / \partial x^{i}$ are differential operators acting on the universal enveloping algebra. For the $\mathfrak{s u}(2)$ case the adjoint representation is given by the defining representation of $\mathfrak{s o}(3)$, that is

$$
\tau_{1}=\left(\begin{array}{ccc}
0 & 0 & 0 \\
0 & 0 & -\mathrm{i} \\
0 & \mathrm{i} & 0
\end{array}\right) \quad \tau_{2}=\left(\begin{array}{ccc}
0 & 0 & \mathrm{i} \\
0 & 0 & 0 \\
-\mathrm{i} & 0 & 0
\end{array}\right) \quad \tau_{3}=\left(\begin{array}{ccc}
0 & -\mathrm{i} & 0 \\
\mathrm{i} & 0 & 0 \\
0 & 0 & 0
\end{array}\right)
$$

Up to the second order in the generators we have then

$$
j^{\frac{1}{2}}(x)=\operatorname{det}^{\frac{1}{2}}\left[\mathbf{1}+\frac{\left(\tau_{i} x_{i}\right)^{2}}{24}+O\left(x^{4}\right)\right]=1+\frac{1}{2} \operatorname{tr} \frac{\left(\tau_{i} x_{i}\right)^{2}}{24}=1+\frac{1}{2} \frac{|x|^{2}}{12}=1+\frac{|x|^{2}}{24}
$$

where we have used

$$
\ln \operatorname{det}(\mathbf{1}+A)=\sum_{1}^{\infty} \frac{(-1)^{n+1}}{n !} \operatorname{tr} A^{n}
$$

When replacing (3.60) in the Duflo isomorphism (3.56) and applying it to polynomials of second order we obtain

$$
\chi_{D}\left(x_{j} x_{k}\right)=I_{P B W} \circ\left(1+\frac{\partial_{i} \partial_{i}}{24}\right)\left(x_{j} x_{k}\right)=I_{P B W}\left(x_{j} x_{k}\right)+\frac{1}{12} \delta_{j k}
$$

When applied to the Casimir function $x_{j} x_{j}$ we finally obtain

$$
I_{P B W}\left(x_{j} x_{j}\right)=X_{j} X_{j}+\frac{1}{4}
$$

where $x_{j} x_{j}$ is an element in the symmetric algebra, that is the symmetric functions on $\mathbb{R}^{3}$ which will be our classical observables, while $X_{j} X_{j}$ indicates a polynomial in the center of the universal enveloping algebra. We then represent the latter as an operator acting on the Hilbert space $\mathfrak{X}=\{|j, m\rangle, j \in \mathbb{N} / 2,-j \leq m \leq j\}$ of the angular momentum eigenstates

$$
\rho: X_{j} X_{j} \rightarrow \hat{X}_{j} \hat{X}_{j}: \mathfrak{X} \mapsto \mathfrak{X}
$$

so that the Duflo quantization prescription for the symmetric function $x_{j} x_{j}$ reads

$$
\hat{\chi}: x_{j} x_{j} \rightarrow \hat{X}_{j} \hat{X}_{j}+\frac{1}{4} \mathbb{I}
$$

with $\hat{\chi}=\rho \circ \chi$ and we obtain for the spectrum

$$
\hat{\chi}\left(x_{j} x_{j}\right)|j m\rangle=\left(j(j+1)+\frac{1}{4}\right)|j m\rangle=\left(j+\frac{1}{2}\right)^{2}|j m\rangle
$$

Let us stress the factor $1 / 4$ which modifies the spectrum. In next section we shall apply this quantization scheme to the hydrogen atom. 


\subsection{The hydrogen atom}

The hydrogen atom belongs to the well known class of Keplerian systems, its reduced Hamiltonian being of the form

$$
H=\frac{p^{2}}{2 m}-\frac{k}{r}
$$

with $m$ the reduced mass of the electron and $k$ the Coulomb constant multiplied by the square of the electron charge. It is well known that the system is super-integrable, with 7 constants of motion, 5 of which being independent. Besides the Hamiltonian, we have the angular momentum

$$
\mathbf{L}=\mathbf{r} \times \mathbf{p}
$$

and the Runge-Lenz vector

$$
\mathbf{A}=\mathbf{p} \times \mathbf{L}-m k \frac{\mathbf{r}}{r}
$$

with

$$
\mathbf{A} \cdot \mathbf{L}=0, \quad \mathbf{A}^{2}=2 m H \mathbf{L}^{2}+m^{2} k^{2}
$$

On considering only negative values for the energy (as we will be interested in the discrete spectrum of the quantum system) we define

$$
\mathbf{D}=\frac{\mathbf{A}}{\sqrt{2} m|E|}
$$

The angular momentum and rescaled Runge-Lenz components close the $\mathfrak{s o}(4)$ Lie algebra w.r.t. the canonical Poisson brackets on $T^{*} \mathbb{R}^{3},\left\{p_{i}, q_{j}\right\}=\delta_{i j}$. We have indeed

$$
\begin{aligned}
\left\{L_{i}, L_{j}\right\} & =\epsilon_{i j k} L_{k} \\
\left\{D_{i}, D_{j}\right\} & =\epsilon_{i j k} L_{k} \\
\left\{L_{i}, D_{j}\right\} & =\epsilon_{i j k} D_{k}
\end{aligned}
$$

On introducing two sets of mutually commuting $\mathfrak{s u}(2)$ generators

$$
\mathbf{B}=\frac{1}{2}(\mathbf{D}+\mathbf{L}), \quad \mathbf{C}=\frac{1}{2}(\mathbf{D}-\mathbf{L})
$$

we have

$$
\mathbf{D}^{2}+\mathbf{L}^{2}=2\left(\mathbf{B}^{2}+\mathbf{C}^{2}\right)=\frac{m k^{2}}{2|E|}
$$

that is, the energy can be expressed in terms of the two quadratic Casimir functions of the $\mathfrak{s o}(4) \simeq \mathfrak{s u}(2) \otimes \mathfrak{s u}(2)$ algebra.

In order to achieve the quantization and obtain the energy spectrum for bounded states we resort to the Duflo quantization map (3.65) and obtain

$$
\hat{\chi}\left(B_{j} B_{j}\right)=\hat{B}_{j} \hat{B}_{j}+\frac{1}{4} \mathbf{I} \quad \hat{\chi}\left(C_{j} C_{j}\right)=\hat{C}_{j} \hat{C}_{j}+\frac{1}{4} \hat{\mathbf{I}}
$$

so that

$$
\hat{\chi}\left(\mathbf{B}^{2}+\mathbf{C}^{2}\right)=\hat{\mathbf{B}}^{2}+\hat{\mathbf{C}}^{2}+\frac{1}{2} \hat{\mathbf{I}}
$$


Resorting to the relation with the Runge-Lenz vector and the angular momentum we find

$$
\hat{\chi}\left(\mathbf{D}^{2}+\mathbf{L}^{2}\right)=\hat{\chi}\left[2\left(\mathbf{B}^{2}+\mathbf{C}^{2}\right)\right]=2\left(\hat{\mathbf{B}}^{2}+\hat{\mathbf{C}}^{2}\right)+\hat{\mathbf{I}}
$$

We then observe that $\mathbf{B}^{2}=\mathbf{C}^{2}$ because of $\mathbf{D} \cdot \mathbf{L}=0$. Therefore

$$
\hat{\chi}\left(\mathbf{D}^{2}+\hat{\mathbf{L}}^{2}\right)|j, m\rangle=\left(4 \hat{\mathbf{B}}^{2}+\hat{\mathbf{I}}\right)|j, m\rangle=(4 j(j+1)+1)|j, m\rangle
$$

Posing $2 j=n$ with $n$ integer and solving for the energy we finally find

$$
|E|=\frac{m k^{2}}{2(n+1)^{2}}
$$

which is the correct result.

\section{The hydrogen atom in the $\star$-quantization approach. Conclu- sions}

In order to compare with the $\star$-quantization approach induced by the star product we have derived in section 2 we have explicitly computed the action of the $\mathfrak{s u}(2)$ Lie algebra generators in the matrix basis in section 2.3. We have found for the Casimir function $x_{0}$

$$
x_{0} \star v_{m \tilde{m}}^{j}=v_{m \tilde{m}}^{j} \star x_{0}=\lambda\left(j+\frac{1}{2}\right) v_{m \tilde{m}}^{j}
$$

so that

$$
x_{0} \star x_{0} \star v_{m \tilde{m}}^{j}=v_{m \tilde{m}}^{j} \star x_{0} \star x_{0}=\lambda^{2}\left(j+\frac{1}{2}\right)^{2} v_{m \tilde{m}}^{j}
$$

This is in fact the symbol of the eigenvalue equation

$$
\hat{\mathbf{X}}^{2}|j, m\rangle=c(j, m)|j, m\rangle
$$

in agreement with the result of the Duflo quantization as in (3.63). Let us notice that, had we chosen a different $\star$-product on $\mathbb{R}^{4}$ than the Moyal product, compatible with the same $\star$ commutation relations for the coordinate functions (1.7), we would have obtained a different result for the quantization of the Casimir function, although inducing the same $\star$-commutation relations for the $\mathbb{R}^{3}$ coordinate functions as in (1.16). For example, the Voros product, in the properly defined matrix basis, $\tilde{v}_{m \tilde{m}}^{j}$ would give [4]

$$
x_{0} \star_{V} \tilde{v}_{m \tilde{m}}^{j}=\tilde{v}_{m \tilde{m}}^{j \star} x_{0}=\lambda j \tilde{v}_{m \tilde{m}}^{j}
$$

and the constant shift is not at all anodyne when the result is applied to the hydrogen atom

spectrum. This is probably due to the fact that only the Moyal quantization is selfdual (meaning that we can use the same operator to map the observables and the states to noncommutative symbols). This suggests that in other quantization schemes we shouldn't use the same matrix basis for the observables and the states as indicated in [13] (also see [34] were the problem of the equivalence of Moyal and Voros quantization is discussed). We shall come back to this issue elsewere. 
To conclude, the Moyal-induced $\star$ product on $\mathbb{R}^{3}$ first introduced in [6] is singled out among other $\star$-quantization schemes in that it agrees with the Duflo quantization map of $\mathfrak{s u}(2)$ Casimir elements. We speculate that the same result will old true for Casimir functions of other three dimensional Lie algebras, whose corresponding Moyal-induced $\star$ products on $\mathbb{R}^{3}$ are illustrated in [6]. Moreover we stress once again that the correct spectrum of quantum systems with a central potential is reproduced in the quantization scheme discussed in the paper.

\section{Acknowledgements}

P. Vitale thanks the Laboratoire de Physique Théorique at Orsay for hospitality while this paper was partially written and acknowledges a grant from the European Science Foundation under the research networking project Quantum Geometry and Quantum Gravity, and partial support by GDRE GREFI GENCO. She also thanks Harald Grosse and Jean-Christophe Wallet for enlightening discussions.

\section{References}

[1] L. Freidel and E. R. Livine, "Effective 3-D quantum gravity and non-commutative quantum field theory," Phys. Rev. Lett. 96, 221301 (2006) arXiv:hep-th/0512113]. A. Baratin and D. Oriti, "Group field theory with non-commutative metric variables," Phys. Rev. Lett. 105, 221302 (2010) arXiv:1002.4723 [hep-th]].

[2] L. Freidel and S. Majid, "Noncommutative harmonic analysis, sampling theory and the Duflo map in 2+1 quantum gravity," Class. Quant. Grav. 25, 045006 (2008) arXiv:hep-th/0601004.

[3] E. Joung, J. Mourad and K. Noui, Three Dimensional Quantum Geometry and Deformed Poincare Symmetry, J. Math. Phys. 50, 052503 (2009) [arXiv:0806.4121 [hep-th]].

[4] Patrizia Vitale, Jean-Christophe Wallet "Noncommutative field theories on $\mathbb{R}_{\lambda}^{3}$ and matrix models" Orsay preprint LPT-12-97.

[5] Patrizia Vitale "Aspects of group field theory" AIP Conference Proceedings 1460 (2012) 101

[6] J. M. Gracia-Bondía, F. Lizzi, G. Marmo and P. Vitale, "Infinitely many star products to play with," JHEP 0204, 026 (2002) arXiv:hep-th/0112092;

[7] J. M. Gracia-Bondía and J. C. Várilly, "Algebras Of Distributions Suitable For Phase Space Quantum Mechanics. 1", J. Math. Phys. 29 (1988) 869.

[8] H. Grosse and R. Wulkenhaar, "Renormalisation of $\phi^{4}$ theory on noncommutative $\mathbb{R}^{2}$ in the matrix base," JHEP 0312 (2003) 019 H. Grosse and R. Wulkenhaar, "Renormalization of phi** 4 theory on noncommutative $\mathrm{R}^{* *} 4$ in the matrix base," Commun. Math. Phys. 256 (2005) 305 hep-th/0401128.

[9] H. Grönewold, "On the Principles of Quantum Mechanics", Physica 12 (1946) 405. 
[10] J. E. Moyal, "Quantum Mechanics as a Statistical Theory," Proc. Cambridge Phil. Soc. 45 (1949) 99.

[11] H. Weyl The theory of Groups and Quantum Mechanics Dover (1931), translation of Gruppentheorie und Quantemmechanik, Hirzel Verlag (1928).

[12] E.P. Wigner, "On the Quantum Correction for Thermodynamic Equilibrium", Phys. Rev. 40 (1932) 749.

[13] V. I. Man'ko, G. Marmo and P. Vitale, "Duality symmetry for star products," Phys. Lett. A 334 (2005) 1. arXiv:hep-th/0407131].

O. V. Man'ko, V. I. Man'ko, G. Marmo and P. Vitale, "Star products, duality and double Lie algebras," Phys. Lett. A 360 (2007) 522. arXiv:quant-ph/0609041.

[14] F. Bayen, M. Flato, C. Fronsdal, A. Lichnerowicz and D. Sternheimer, "Deformation theory and quantization. I. Deformation of symplectic structures", Ann. Phys. (NY) 111 (1978) 61; "Deformation theory and quantization. II. Physical applications", Ann. Phys. (NY) 111 (1978) 111.

[15] A. Zampini, "Applications of the Weyl-Wigner formalism to noncommutative geometry," arXiv:hep-th/0505271.

[16] R. Estrada, J. M. Gracia-Bondia and J. C. Varilly, "On Asymptotic expansions of twisted products," J. Math. Phys. 30 (1989) 2789.

[17] A. Voros, "Wentzel-Kramers-Brillouin method in the Bargmann representation", Phys. Rev. A 406814 (1989).

[18] V. Gayral, J. M. Gracia-Bondia, B Iochum, T. Schücker and J. C. Varilly, Moyal planes are spectral triples, Comm. Math. Phys. 246 (2004) 569.

[19] V. I. Manko, G. Marmo, P. Vitale and F. Zaccaria, "A Generalization of the JordanSchwinger map: Classical version and its q deformation," Int. J. Mod. Phys. A 9, 5541 (1994) hep-th/9310053.

[20] A. B. Hammou, M. Lagraa and M. M. Sheikh-Jabbari, "Coherent state induced star product on $\mathrm{R}^{* *} 3$ (lambda) and the fuzzy sphere," Phys. Rev. D 66, 025025 (2002) arXiv:hep-th/0110291.

[21] S. Galluccio, F. Lizzi and P. Vitale, "Twisted Noncommutative Field Theory with the WickVoros and Moyal Products," Phys. Rev. D 78 (2008) 085007 [arXiv:0810.2095 [hep-th]].

[22] S. Galluccio, F. Lizzi and P. Vitale, "Translation Invariance, Commutation Relations and Ultraviolet/Infrared Mixing," JHEP 0909 (2009) 054 [arXiv:0907.3640 [hep-th]].

[23] A. Tanasa and P. Vitale, A. Tanasa and P. Vitale, "Curing the UV/IR mixing for field theories with translation-invariant * products," Phys. Rev. D 81, 065008 (2010) arXiv:0912.0200 [hep-th]]. 
[24] J. M. Gracia-Bondía and J. C. Várilly, "The Moyal representation for spin" Annals of Physics 190, 107 (1989)

H Figueroa, J. M. Gracia-Bondía and J. C. Várilly, "Moyal quantization with compact symmetry groups and noncommutative harmonic analysis" Journal of Mathematical Physics 31, 2664 (1990)

[25] O. V. Man'ko, V. I. Man'ko and G. Marmo, "Star product of generalized Wigner-Weyl symbols on $\mathrm{SU}(2)$ group, deformations, and tomographic probability distribution," Phys. Scripta 62, 446 (2000).

[26] S. Mancini, V. I. Man'ko and P. Tombesi, "Symplectic tomography as classical approach to quantum systems," Phys. Lett. A 213, 1 (1996) quant-ph/9603002.

[27] V. I. Manko, L. Rosa and P. Vitale, "Probability representation in quantum field theory," Phys. Lett. B 439, 328 (1998) hep-th/9806164.

V. I. Man'ko, L. Rosa and P. Vitale, "Time dependent invariants and Green functions in the probability representation of quantum mechanics," Phys. Rev. A 57, 3291 (1998) quant-ph/9802030.

[28] G. Marmo, P. Vitale and A. Zampini "Noncommutative differential calculus for Moyal subalgebras" J. Geom. Phys. 56 (2006) 611 arXiv:hep-th/0411223

[29] F. Lizzi, P. Vitale and A. Zampini, "The Fuzzy disc," JHEP 0308 (2003) 057 hep-th/0306247]. "The Beat of a fuzzy drum: Fuzzy Bessel functions for the disc," JHEP 0509 (2005) 080 hep-th/0506008. "The fuzzy disc: A review," J. Phys. Conf. Ser. 53, 830 (2006). "From the fuzzy disc to edge currents in Chern-Simons theory," Mod. Phys. Lett. A 18, 2381 (2003) hep-th/0309128.

[30] M. Duflo, Opérateurs différentiels bi-invariants sur un groupe de Lie. Ann. Sc.Ec. Norm. Sup. 10 (1977), 107-144.

[31] Damien Calaque, Carlo A. Rossi "Lectures on Duflo Isomorphisms in Lie Algebra and Complex Geometry" (EMS Series of Lectures in Mathematics) (2011)

[32] M. Kontsevich, "Deformation quantization of Poisson manifolds, I", Lett. Math. Phys. 66 (2003) 157. arXiv:q-alg/9709040.

[33] K. Noui, A. Perez and D. Pranzetti, "Canonical quantization of non-commutative holonomies in 2+1 loop quantum gravity," JHEP 1110 (2011) 036 [arXiv:1105.0439 [gr-qc]].

$[34]$

[35] P. Basu, B. Chakraborty and F. G. Scholtz, "A Unifying perspective on the Moyal and Voros products and their physical meanings," J. Phys. A A 44, 285204 (2011) arXiv:1101.2495 [hep-th]]. 